\title{
ESTRATEGIAS DIDÁCTICAS PARA EL APRENDIZAJE DE LA CONTADURÍA PÚBLICA
}

Milton García Barbosa

Universidad de Cartagena

Colombia

Deilo Martínez Maza

Universidad de Cartagena

Colombia 
Panorama Económico, Vol. 27 - No. 1 (Enero - Marzo de 2019), pp. 290-309

Milton García Barbosa

Deilo Martínez Maza

JEL: A20, A22, A29

\section{Estrategias didácticas para el aprendizaje de la Contaduría Pública}

\section{Resumen}

El trabajo de investigación tiene como propósito proponer un modelo de proyecto de aula que articule la investigación con los contenidos curriculares de la disciplina de las ciencias contables, para potenciar la aprehensión de los conocimientos disciplinares de la profesión contable en los estudiantes y fomentar una cultura de investigación de cara a los desarrollos cognitivos que requieren del egresado los mercados, producto de la globalización y las nuevas tendencias de la profesión contable, propios de la arquitectura financiera internacional. La importancia de la investigación radica en que se asume el proyecto de aula como un proceso, completo, intencional y consciente (Rincón, 2012), en donde el estudiante como actor principal, aprende a partir de la formulación de problemas que articulan las áreas disciplinares de conocimiento de su plan curricular y porque además, explora las diferentes concepciones que sobre los proyectos de aula se han esgrimido para enfatizar en un modelo que se adecue a las necesidades de los Programas de Contaduría Pública. Los resultados del trabajo muestran que la estrategia de articular los trabajos de Aprendizaje Basado en Problemas (ABP) realizados por los estudiantes al proyecto de aula, propicia la aprehensión de conocimientos en los estudiantes y la generación de producción intelectual de los docentes.

Palabras clave: Aprendizaje, aula, proyecto, modelo pedagógico.

\section{Stratégies didactiques pour l'apprentissage de la Comptabilité}

\section{Résumé}

Le travail de recherche vise à proposer un modèle de projet de classe qui articule la recherche avec le contenu curriculaire de la discipline des sciences comptables, à améliorer l'appréhension des connaissances disciplinaires de la profession comptable chez les étudiants et à favoriser une culture de la recherche face à la les développements cognitifs dont le diplômé a besoin sur les marchés, le produit de la mondialisation et des nouvelles tendances de la profession comptable, typiques de l'architecture financière internationale. L'importance de la recherche réside dans le fait que le projet de classe est supposé comme un processus complet, intentionnel et conscient (Rincón, 2012), où l'étudiant en tant qu'acteur principal, apprend de la formulation de problèmes qui articulent la connaissance des domaines disciplinaires de son plan du curriculum et aussi parce qu'il explore les différentes conceptions que les projets en classe ont été utilisés pour mettre l'accent dans un modèle qui répond aux besoins des programmes de comptabilité publique. Les résultats des travaux montrent que la stratégie d'articulation du travail d'apprentissage par problèmes (ABP) mené par les élèves au projet de classe, favorise l'appréhension des connaissances chez les élèves et la génération de la production intellectuelle des enseignants.

Mots clés: Apprentissage, salle de classe, projet, modèle pédagogique.

\section{Didactic strategies for learning Accounting}

\section{Abstract}

The research work aims to propose a classroom project model that articulates the research with the curricular contents of the discipline of accounting sciences, to enhance the apprehension of the disciplinary knowledge of the accounting profession in students and foster a culture of Research in the face of the cognitive developments that the graduate requires in markets, the product of globalization and new trends in the accounting profession, typical of the international financial architecture. The importance of research lies in the fact that the classroom project is assumed as a complete, intentional and conscious process (Rincón, 2012), where the student as the main actor, learns from the formulation of problems that articulate the disciplinary areas knowledge of its curriculum plan and also because it explores the different conceptions that classroom projects have been used to emphasize in a model that meets the needs of Public Accounting Programs. The results of the work show that the strategy of articulating the work of Problem Based Learning (ABP) carried out by the students to the classroom project, fosters the apprehension of knowledge in the students and the generation of intellectual production of the teachers.

Key words: Learning, Classroom, Project, Pedagogical model. 


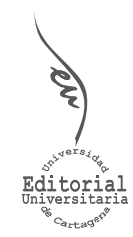

Artículo de Investigación

\section{Estrategias didácticas para el aprendizaje de la Contaduría Pública}

INFORMACIÓN DEL ARTíCULO

Recepción del artículo: 06/07/2018

Concepto de evaluación: 01/09/2018

Aceptación del artículo: 05/10/2018
Milton García Barbosa*

Universidad de Cartagena, Colombia

Deilo Martínez Maza

Universidad de Cartagena, Colombia

\section{INTRODUCCIÓN}

Las estrategias pedagógicas son las actividades, acciones y tareas que desarrollan los profesores para facilitar el proceso de enseñanza-aprendizaje de las diferentes disciplinas que desarrollan los estudiantes durante el transcurso de su formación profesional. La forma como se construye y apropia el conocimiento, se relaciona directamente con el modelo pedagógico que desarrolla la institución fundamentado en:

"El aprendizaje entendido como el proceso de utilización y comisión de las habilidades de pensamiento científico en estrategias para asimilar y acomodar nuevos conocimientos; con el cual se busca desarrollar vitalmente las habilidades, destrezas, técnicas, tecnológicas, y representaciones que componen el eje fundamental del perfil profesional e igualmente, desarrollar herramientas novedosas frente a problemas específicos como perspectiva de intervención práctica y puntual" (Universidad del Sinú, 2003, p. 20).

En este sentido, para el desarrollo de su modelo pedagógico la institución propugna por la formación de un profesional con las competencias necesarias para proyectarse internacionalmente y capacidad para desarrollarse en el campo de las ciencias económicas. El modelo pedagógico establece las estrategias y lineamientos para que, en el desarrollo del currículo, se apropien desde la investigación los conocimientos que permitan al estudiante la aprehensión asertiva de los conocimientos, y en ese sentido, la metodología del A.B.P. (Aprendizaje Basado en Problemas) se constituye en la apuesta básica para el desarrollo de las competencias y habilidades del discente.

Los procesos investigativos en la disciplina contable son en la actualidad muy limitados y se puede decir, que no se priorizan en los procesos de formación, tanto en el estudiante como el profesor, no adoptan la investigación como el fundamento para la aprehensión

\footnotetext{
*. Docente de carrera del Programa de Contaduría Pública de la Universidad de Cartagena. Autor de correspondencia: mgarciab1@unicartagena.edu.co
} 
de los conocimientos y el desarrollo de una cultura investigación al interior de las Escuelas, lo cual se ve reflejado en los menor actividad investigativa (mínima producción investigativa, grupos y semilleros de investigación invisibilizados a nivel nacional e internacional).

De allí la necesidad de desarrollar una estrategia que permita en la formación profesional, articular la metodología del A.B.P. desarrollados en las diferentes disciplinas del currículo con los proyectos de aula, para que el aula de clase se convierta en escenarios donde los estudiantes aprendan a construir sus propios conocimientos a partir de problemas concretos de las disciplinas que se desarrollan en ella.

\section{MARCO TEÓRICO}

La producción de conocimiento se asocia con el proceso universitario, y en este sentido, se podría decir, no es un proceso lineal. Los impactos en el conocimiento necesitan de un personal con una profunda formación pedagógica y disciplinal, asociada a los espacios (infraestructura) en donde se pueda desarrollar conocimiento, que son los laboratorios de investigación y desarrollo. Estos pueden estar dentro de la institución o en centros de investigación. Pero nada de eso es posible si no tenemos una educación superior que este en el proceso continuo de generar un mejor recurso humano formado.

Si bien es cierto que la educación superior ha tenido como áreas misionales la docencia, la investigación y la extensión, y ahora, la internacionalización, la tendencia es, a que la investigación se convierta en un sinónimo de calidad en el para las instituciones de educación superior, al tiempo que aporta conocimientos aplicables a la solución de las problemáticas y al desarrollo de los países.

\section{Teorías del aprendizaje e investigación formativa.}

Las teorías pedagógicas nos muestran que las diferencias entre los que aprenden y en el entorno donde reciben su formación, afectan el aprendizaje de los estudiantes, aunque se podría decir que no es taxativo. El rol que juega el entorno ambiental, es en sí, muy importante, para la estimulación y la forma como se fortalecen las respuestas del aprendiz.

De acuerdo con Schunk (2012),

Las teorías conductuales asignan menos importancia a las diferencias del aprendiz que las teorías cognoscitivas. Dos variables del estudiante que considera el conductismo son: el historial de reforzamiento (el grado al que el individuo ha sido reforzado en el pasado por desempeñar la misma tarea o una similar) y el estadio de desarrollo en que se encuentra (p.401).

Las teorías cognoscitivas hacen énfasis en la influencia que tiene las condiciones ambientales en el proceso de aprendizaje del estudiante, se aprende más de las motivaciones que ofrece el entorno (Bruning, Schraw, Norby, \& Ronning, 2004) en esto el docente juega un papel importante al proporcionarle los datos de entrada. 
Iniciemos por el constructivismo, un modelo en el que tanto estudiantes como docentes construyen su conocimiento de manera autónoma y con sus ritmos. El constructivismo se soporta en un gran porcentaje en la teoría sobre el desarrollo humano desarrollada por Piaget y Vygotsky.

Los teóricos del constructivismo rechazan no aceptan la teoría de que existen verdades científicas y se apegan más al descubrimiento y la verificación; plantean que no todas las afirmaciones se puedan entender como verdadera, y que, en vez de eso, se deben observar con sentido crítico. Ninguna teoría posee la verdad absoluta y de allí, que de diferentes formas podamos, mentalmente, construir el mundo.

La teoría de Vygotsky tiene un fundamento fuerte en la relación de las circunstancias sociales, históricas, culturales e individuales, como esenciales para el desarrollo personal (Tudge y Scrimsher, 2003). La interacción de los grupos de aprendizaje y de las personas en los ambientes entórnales, estimulan la evolución y el crecimiento cognoscitivo. Para Vygotsky estas interacciones son importantes porque le permite al aprendiz transformar sus pensamientos.

Otro aspecto, lo constituye la enseñanza para el descubrimiento cuyo fundamento está basado en el planteamiento de preguntas, situaciones problemáticas o fases difíciles de resolver y motivar a los estudiantes a formular preguntas cuando tienen desconocimiento de las cosas

Para inducir a los aprendices en la construcción del conocimiento autónomo, y a generar discusiones en el aula de clases, los profesores deben plantearles preguntas de razonamiento y especificarles que la actividad no constituye una evaluación, para suavizar las condiciones del entorno en el aprendizaje. Cuando los aprendices no están habituados a la metodología del aprendizaje por descubrimiento, es cuando más se requiere de estructura por parte del profesor. (Schunk, 2012).

Uno de los métodos y prácticas poderosas para la investigación formativa es el ABP: Aprendizaje Basado en Problemas, que vinculado a la educación superior es un ingrediente importante para la comprensión de los problemas de la sociedad. El ABP sirve en el pregrado de forma virtuosa, favoreciendo la interdisciplinariedad, el dialogo de saberes, propio de la investigación moderna.

\section{Los Proyectos de Aula}

El concepto de proyecto de aula toma fuerza como propuesta pedagógica durante el siglo XX a partir de la perspectiva socio histórico y cultural de Vygotsky, cuando plantea la enseñanza no como un proceso de transmisión de conocimientos, sino que el estudiante construye su propio conocimiento y aprende a construirlo, a partir de la forma en que "percibe la realidad, la organiza y le da sentido en forma de constructos, gracias a la actividad de su sistema nervioso central" como plantea (Ortiz, 2015, p. 5).

Los proyectos de aula:

Constituyen un planteamiento sobre el conocimiento escolar, vinculado a una concepción en la que se da importancia no sólo a la adquisición de estrategias cognitivas de orden superior, sino al papel del estudiante como responsable de su propio aprendizaje en relación con un replanteamiento del saber escolar. 
Esto supone aprender a investigar un tema desde un enfoque relacional que une ideas claves y metodologías de diferente disciplina (Rincón, 2012, p. 23).

Lo anterior nos lleva a entender que los proyectos de aula son una estrategia pedagógica que involucra a docentes y estudiantes, quienes, a través de un trabajo colaborativo de contribución y participación, aprehenden los conocimientos.

\section{MÉTODOS}

Para el logro de los objetivos, las actividades y la captación de la información se realizaron a través del método deductivo con un enfoque cualitativo-descriptivo, en razón a que su propósito fue caracterizar un modelo de proyecto de aula de la disciplina contable de la Universidad del Sinú, producto del análisis de las tendencias actuales respecto de esta estrategia metodológica. La metodología propuesta se dividió en tres fases:

Primera fase: revisión analítica de las teorías desarrolladas sobre la metodología de los proyectos de aula.

Segunda fase: Revisadas y analizadas las teorías relacionadas con los proyectos de aula, se procedió a estudiar su aplicación.

Tercera fase: recolección y procesamiento de la información, para establecer un modelo de proyecto de aula que se adecue a las necesidades del Programa.

\section{RESULTADOS}

De acuerdo con el Proyecto Educativo Institucional, la Universidad ${ }^{1}$ propende por formar personas capaces de organizar, planificar, construir y apropiarse del conocimiento científico, tecnológico, filosófico y ético, potencializando una cultura que a partir de sus saberes y valores aporte al desarrollo económico-social del país. Concibe al ser como un hombre íntegro, sabio y útil a la sociedad a través de su desempeño social y en este sentido, los valores que sustentan la formación de sus estudiantes se sustentan en "el respeto a la vida y los derechos humanos inmersos en una cultura regional con conciencia global” (Universidad del Sinú, p. 11).

De acuerdo con el P. E. I. el currículo se "concibe como una construcción social en donde la pertinencia del conocimiento y su puesta en contexto son elementos que permiten su significación y su validez" (Universidad del Sinú, p. 28).

\section{El modelo pedagógico}

El modelo pedagógico está enfocado hacía el aprendizaje significativo, entendido como "el proceso de utilización y comisión de las habilidades de pensamiento crítico en estrategias para asimilar y acomodar nuevos conocimientos" (Unisinú, p. 24). En donde el currículo tiene como ejes fundamentales: la cultura de la participación en función de la construcción de conocimiento autónomo y la dimensión de 
investigación que permiten la integración del contexto científico con la construcción de conocimientos pertinentes, acordes con el contexto político, social, económico y financiero nacional e internacional.

\section{Políticas para la investigación}

El Proyecto Educativo Institucional establece las políticas para la investigación, de las cuales muestra gran importancia para el desarrollo de la academia aquella en que se hace necesario que los programas curriculares articulen diferentes áreas y líneas que permita definir el trabajo académico del estudiante, y propicie la capacidad de indagación y búsqueda que generen en el disicente la formación de un espíritu científico.

Con base en lo anterior, la Universidad definió el desarrollo de A.B.P. (Aprendizaje Basado en Problemas) en sus programas de pregrado como estrategia que dinamice no solo el aprendizaje autónomo del estudiante, sino también, la cultura de investigación al interior de las unidades académicas (Vizcarro \& Juárez, 2008) que se vean reflejadas en productos de investigación de los docentes, como posters y presentación de ponencias en eventos científicos, artículos científicos en revistas indexadas del nivel nacional e internacional.

\section{Enfoques metodológicos del proyecto de aula}

El aspecto investigativo se constituye en una problemática de la educación contable del país, y representa una realidad de la Escuelas de Contaduría Pública. Ante esta situación las instituciones pueden contemplar en sus modelos pedagógicos, estrategias y metodologías como el proyecto de aula, para inducir a profesores y discentes en su formación investigativa, buscando una mayor dinámica en la producción científica y en la contextualización del conocimiento. Su aplicación difiere entre instituciones, y en algunos casos, la metodología solo se instaura en la primera fase de formación profesional, dejando de lado los últimos semestres de formación profesional.

La Investigación se plantea a través de la asignatura de Seminarios de Investigación que se desarrolla de manera práctica bajo la premisa "se aprende a investigar investigando". De esta manera, en primer semestre se consolidan las bases sobre la epistemología de los paradigmas del conocimiento y sus teorías. En segundo semestre, aprende a identificar problemas de investigación y a justificar la realización de un proyecto. En tercer semestre, realiza abordajes teóricos y conceptuales para comprender los fenómenos y problemáticas identificadas en las empresas, planteando el diseño metodológico pertinente con la investigación realizada: recoge, organiza y analiza la información necesaria de manera apropiada y pertinente. En cuarto semestre, plantea conclusiones, formula recomendaciones y diseña un plan de transformación pertinente. En quinto semestre, se concreta la Propuesta de Investigación que se fortalece, estructura y consolida con los conocimientos específicos que va construyendo en el proceso de formación profesional (sexto, séptimo, octavo y noveno semestre) como Contador Público. Finalmente, en decimo semestre, realiza su proyecto de grado y socializa los resultados obtenidos. 


\section{El proyecto de aula como estrategía pedógógica en Contaduría Pública}

El modelo educativo para el aprendizaje de la contabilidad se ha erigido sobre la base de la asimilación de verdades y contenidos temáticos (asignaturales) que le son transmitidos al estudiante a través de una delimitación de los objetivos, que en esencia no le permiten analizar e interpretar críticamente las realidades del acervo contable dentro de su contexto en específico. Esto obedece a un pensamiento generalizado hasta hace menos de 10 años, en donde, se afirmaba erróneamente que el aprendizaje tenía su residencia en la transmisión de conocimientos y en donde el docente ejercía gran autoridad, y que en la práctica terminó induciéndolo al rol de técnico educativo (Deleuze y Guattari, 2001). En el fondo, el modelo lo que pretende es que tanto estudiantes como profesores adopten el proceso de aprendizaje con nuevos y mejores procedimientos y técnicas, cual ha sido el decantar de la educación contable en la ciudad de Cartagena a través de su historia, de allí la baja o poca producción científica de los profesionales contables.

En la enseñanza contable y de acuerdo a la problemática de la educación contable del país, los esfuerzos de las administraciones educativas han llevado al establecimiento de propuestas educativas con tendencias a resolver las deficiencias del modelo educacional. En algunos casos, se contempla en el modelo pedagógico estrategias y metodologías como el proyecto de aula y el A.B.P. para inducir tanto al estudiante y al docente en los procesos de formación investigativa buscando una mayor dinámica en la producción científica y en la aprehensión de los conocimientos disciplinares.

\section{El aprendizaje basado en problemas (A.B.P.) Como una metodología para el desarrollo de los proyectos de aula}

El A.B.P. es un método de enseñanza-aprendizaje centrado en el estudiante, formulado por la escuela de medicina de la Universidad de McMaster (Canadá) en la década de los setenta y desarrollada luego en Europa por la Universidad de Maastricht (Instituto Tecnológico y de Estudios Superiores de Monterrey (ITESM), 2018), el modelo de los siete (7) pasos adoptado en la Universidad del Sinú (Moust, Bouhuijs y Schmidt, 2007), cuyo objetivo es formar estudiantes que a través del autoaprendizaje y con la orientación o tutoría de un docente-tutor, sean capaces de estudiar y analizar en su propio contexto las diferentes problemáticas a las que se va a enfrentar en el campo laboral.

Las universidades implementan el A.B.P. dentro de su política de formación integral, basada en el concepto de créditos académicos. A manera de ejemplo, un (1) crédito académico equivale a cuarenta y ocho (48) horas totales por semestre (Ministerio de Educación Nacional, 2002), diez y seis (16) de trabajo presencial y treinta y dos (32) de trabajo independiente del estudiante, así: 
Tabla 1.

Relación horas de trabajo presencial e independiente

\begin{tabular}{c|c|c|c}
\hline Créditos & $\begin{array}{l}\text { Horas de trabajo } \\
\text { presencial por } \\
\text { semestre }\end{array}$ & $\begin{array}{l}\text { Horas de trabajo } \\
\text { independiente } \\
\text { por semestre }\end{array}$ & $\begin{array}{l}\text { Horas totales } \\
\text { por crédito en } \\
\text { el semestre }\end{array}$ \\
\hline 1 & 16 & 32 & 48 \\
2 & 32 & 64 & 96 \\
3 & 48 & 96 & 144 \\
4 & 64 & 128 & 192 \\
5 & 80 & 160 & 240 \\
\hline
\end{tabular}

Fuente: Política A.B.P. Unisinú

La dinámica del método A.B.P. requiere que el trabajo investigativo del estudiante, esté orientado por un docente-tutor que planifique y direccione su proceso de aprendizaje autónomo (Vieira, 2003). Esta característica se ve plasmada en los lineamientos definidos por la institución para insertar los tiempos que se deben dedicar a la metodología dentro del tiempo de trabajo presencial dedicado por el docente. (Ver Tabla $\mathrm{N}^{\circ}$ 2).

Tabla 2.

Relación de horas de A.B.P. por crédito

Asignatura de 1 crédito

Asignatura de 2 créditos

Asignatura de 3 créditos
3 horas de A.B.P. por semestre

6 horas de A.B.P. por semestre

9 horas de A.B.P. por semestre

Fuente: Política A.B.P. Unisinú

Se pretende con ello, que el docente dentro de su tiempo programado para el desarrollo de su asignatura, aplique la metodología y para ello requiere de una planeación adecuada de los tiempos para facilitar el trabajo investigativo del estudiante (Porlan, 1990).

La estructura metodológica del A.B.P. en la institución se articula a ejes temáticos aglutinados en varias asignaturas interrelacionadas inter y multi disciplinariamente, intenciona el cambio en la manera de acceder al conocimiento por parte del estudiante.

La implementación de los siete (7) pasos sigue los siguientes parámetros: 
Tabla 3.

A.B.P. por asignaturas de acuerdo con los créditos.

\begin{tabular}{|c|c|c|c|c|}
\hline Sesiones & Pasos de A.B.P. & Actividad & TP & TI \\
\hline 1 Sesión & $\begin{array}{l}\text { 1. Lectura del problema. } \\
\text { 2. Lista de lo que se } \\
\text { conoce y desconoce. } \\
\text { 3. Lluvia de ideas. }\end{array}$ & $\begin{array}{l}\text { Salón de clase, } \\
\text { tutoría del docente. }\end{array}$ & $1 \mathrm{H}$ & $2 \mathrm{H}$ \\
\hline 2 Sesión & $\begin{array}{l}\text { 4. Clasificación de ideas. } \\
\text { 5. Definición del problema } \\
\text { y objetivos de aprendizaje. } \\
\text { 6. Investigación, obtención } \\
\text { de información. }\end{array}$ & $\begin{array}{c}\text { Trabajo independiente } \\
\text { Búsqueda en biblioteca, } \\
\text { Base de datos }\end{array}$ & $1 \mathrm{H}$ & $2 \mathrm{H}$ \\
\hline 3 Sesión & $\begin{array}{l}\text { 7. Generar conclusiones } \\
\text { y presentar resultados. }\end{array}$ & Presentación final & $1 \mathrm{H}$ & $2 \mathrm{H}$ \\
\hline
\end{tabular}

Fuente: Política A.B.P. Unisinú

De lo cual se desprende que, en una asignatura que tiene un (1) crédito académico el estudiante debe desarrollar un (1) trabajo de A.B.P.; si tiene dos (2) créditos, desarrolla dos (2) A.B.P.; y si tiene tres (3) entonces hay que desarrollar tres (3) A.B.P., todos ellos con la tutoría del docente y planificado en conjunto en el aula.

Lo ideal, para alcanzar los objetivos de la política propuesta por la institución, es superar el concepto asignaturista y traducirlas en módulos que contengan el conjunto de asignaturas propuestas en la malla curricular, que apunten a la solución de problemas sociales, políticos y económicos significativos (Cerda, 2003).

\section{El proyecto de aula como estrategia para el aprendizaje en la Contaduría Pública}

La educación contable en Colombia es pragmática con un profundo enfoque técnicoinstrumentalista con sujeción a la norma (Martínez, 2008)., y aunque las propuestas educativas de los programas de Contaduría Pública enfatizan en una formación integral, perfilando profesionales con sentido crítico, la experiencia muestra un profesional cuasi experto en la norma, formado para el mundo del trabajo, pero con bajísimos niveles de comprensión y de análisis crítico de las problemáticas que afectan tanto a las empresas, como a la profesión contable del país. Esta situación que está inserta en la cultura educacional de la profesión, es posible dinamizarla, de tal manera que se pueda entender a partir del aprendizaje significativo, la técnica en el plano de la praxis de la contabilidad como señala (Farias \& De Agüero, 2014), pero con una propensión holística, en el campo social, cultural y político necesarios en el aprendizaje de la Contaduría Pública. 
De acuerdo con (Londoño y Calvache, 2010), el proyecto de aula es concebido como una metodología aplicada en el aula mediante la cual los conocimientos de las unidades de aprendizaje se incorporan en el proceso a la solución de problemas partiendo de un proyecto, utilizando las estrategias didácticas que le permitan al estudiante acceder autónomamente al conocimiento, y a adquirir las competencias y habilidades necesarias en su proceso de formación. El aula cambia de significado y se convierte en un escenario, donde un grupo de personas se encuentran para interactuar y discutir en torno a un conocimiento (Silva y Maturana, 2017).

\section{Estructura del proyecto de aula en la escuela de Contaduría Pública de la Universidad del Sinú}

La propuesta metodológica de articulación del A.B.P. al proyecto de aula (Chaparro, 2003), parte de la planeación académica estructurada por la dirección de la escuela. La aplicación de la política se basa en el desarrollo de los A.B.P. en las asignaturas disciplinares por semestres. Lo anterior implica que, si en un semestre se desarrollan 4 (cuatro) asignaturas de dos (2) créditos cada una, hay que realizar ocho (8) A.B.P. que van a sustentar el desarrollo del proyecto de aula.

La estrategia pretende la aplicación de la metodología del proyecto de aula a partir del trabajo investigativo que realizan los estudiantes a través de los A.B.P. que son la exigencia producto de la política académica de la universidad y obliga al dialogo permanente de los docentes en el propósito de estructurar el proyecto.

Con base en lo anterior, el proyecto de aula tendrá la siguiente estructura:

\section{Operacionalización del proyecto}

Los proyectos de aula son el producto de la planificación y su desarrollo. Los proyectos de aula obedecen al eje temático establecido en la planeación académica, articulados a los A.B.P. que se realizan en cada una de las asignaturas disciplinares. En este sentido, en cada semestre una asignatura disciplinar se erige como la base para desarrollar los ejes temáticos y por consiguiente, el docente se configura como el líder del proyecto.

En cada una de estas etapas se debe realizar un mínimo de actividades, que se relacionan a continuación.

\section{Planificación y desarrollo}

La planificación del proyecto de aula es prioridad de los docentes e incluye:

Seleccionar las competencias. Los docentes de las asignaturas disciplinares seleccionan las competencias que desarrollarán los estudiantes, generales y específicas. Se socializa con los estudiantes las habilidades y destrezas que éste debe desarrollar con el proyecto de aula.

Plantear el problema. Una vez seleccionadas las competencias se diseña la problemática que debe abordar el estudiante para la presentación de los productos. El problema puede surgir de una pregunta problema o del desarrollo 
de un estudio de caso, y se hace necesaria su sistematización a través de subpreguntas que direccionen las tareas que realizarán los estudiantes.

Organizar los equipos de trabajo. Se conformarán equipos de trabajo para realizar las tareas requeridas en el proyecto, que de acuerdo con la complejidad del problema tendrán un mínimo de 4 estudiantes y un máximo de 8 y su elección es prioridad del estudiante. La planificación de las tareas a desarrollar por los estudiantes debe responder a las preguntas: ¿cómo se llama el proyecto?, ¿qué y cómo se hará?, ¿cuál es el tiempo de entrega de los productos?, ¿cuáles son las responsabilidades de cada uno de los miembros del equipo?, y ¿qué recursos se necesitan para desarrollar esas tareas?

Diseñar las herramientas de evaluación. Para cada fase del proyecto de aula, se diseñan las herramientas que le permita a los docentes controlar el trabajo de campo que realiza el estudiante y que le permita la retroalimentación para el mejoramiento del proceso de aprendizaje (Tobón, Pimienta y García, 2010)

\section{Materialización del proyecto de aula}

La responsabilidad del proyecto de aula es compartida entre docentes y estudiantes, de acuerdo con los roles que cumplen cada uno. Sin embargo, y atendiendo al concepto del docente como guía y dinamizador del proceso de aprendizaje del estudiante, la mayor responsabilidad del éxito recae en el docente.

Hay una serie de exigencias académicas y de investigación al docente desde la dirección académica que hacen del proyecto docente la herramienta adecuada para desarrollarlas, por ejemplo, cada proyecto de aula debe generar un (1) artículo de revisión publicable por cada asignatura que lo integra.

Para materializar la propuesta, se presenta a manera de guía, la forma en que quedaría estructurado el proyecto de aula de segundo semestre.

Tabla 4.

Cuadro de asignaturas de $2^{\circ}$ semestre.

\begin{tabular}{l|c|c|c|c}
\hline \multicolumn{2}{c}{ SEGUNDO SEMESTRE } & \\
Asignaturas & $\begin{array}{c}\text { \# de créditos } \\
\text { académicos }\end{array}$ & $\begin{array}{c}\text { Trabajo } \\
\text { presencial }\end{array}$ & $\begin{array}{c}\text { Trabajo } \\
\text { ind.* }\end{array}$ & $\begin{array}{c}\text { Número de A.B.P. } \\
\text { (Aprendizaje Basado } \\
\text { en Problemas) }\end{array}$ \\
\hline Business english II & 2 & 2 & 4 & 0 \\
Cálculo & 3 & 3 & 6 & 0 \\
Costos para la toma de decisiones & 3 & 3 & 6 & 3 \\
Procesos contables & 3 & 3 & 6 & 3 \\
Ética general y convivencia ciudadana & 2 & 2 & 4 & 0 \\
Legislación societaria & 2 & 2 & 4 & 0 \\
Emprendimiento II & 2 & 2 & 4 & 0 \\
Seminario investigativo II & 2 & 2 & 4 & 8 \\
\hline TOTALES & 19 & 19 & 38 & 0
\end{tabular}

Fuente: Elaboración propia 
De acuerdo con la Tabla No.2 las asignaturas disciplinares son: Procesos contables, Costos para la toma de decisiones y Legislación societaria, lo que implica el desarrollo de ocho (8) A.B.P. que se articulan con el proyecto de aula de segundo semestre. A continuación se expone un ejemplo-guía del respectivo proyecto.

La asignatura líder es PROCESOS CONTABLES, la cual propicia toda la base teóricapráctica del proyecto y las asignaturas de apoyo son: Costos para la toma de decisiones y Legislación societaria, que contribuyen con su desarrollo en todas sus fases.

Tabla 5.

Proyecto de aula $2^{\circ}$ semestre

\begin{tabular}{|l|l|l|}
\hline \multirow{2}{*}{} & $\begin{array}{l}\text { PROGRAMA } \\
\text { CONTADURÍA PÚBLICA }\end{array}$ & Fódigo: \\
\cline { 3 - 3 } & Fecha: \\
\cline { 2 - 3 } & PROYECTO DE AULA & Aprobado por: \\
\hline
\end{tabular}

\section{TÍTULO:}

PROYECTO DE AULA $2^{\circ}$ SEMESTRE:

\section{COMPETENCIAS ESPECÍFICAS A DESARROLLAR:}

-

\section{GENÉRICAS}

1. Aprendizaje: aprende, analiza y sintetiza los conceptos de contabilidad general y contabilidad de costos.

2. Gerenciales: desarrolla las capacidades para ejercer liderazgo, organizar, trabajar autónomamente y planear el trabajo de forma tal que le permita tomar decisiones, solucionar problemas y generar ideas innovadoras.

\section{- $\quad$ ESPECÍFICAS}

1. Aprehensión y comprensión de los conceptos de contabilidad general y de costos en contextos globales.

2. Entiende el proceso contable para la contabilización de los hechos económicos relacionados con la contabilidad general y de costos.

3. Se apropia de las normas que en Colombia están vigentes para los comerciantes y el comercio en general que respaldan el proceso contable.

HABILIDADES A DESARROLLAR POR EL ESTUDIANTE:

1. Realiza el proceso de contabilización de las transacciones relacionadas con la contabilidad general y de costos en los libros de contabilidad.

2. Efectúa análisis de estados financieros y de costos para la toma de decisiones.

3. Realiza los análisis de flujos de efectivo y presupuestos de costos.

4. Análisis de la legislación comercial en Colombia. 
Tabla 5.

\section{Proyecto de aula $2^{\circ}$ semestre}

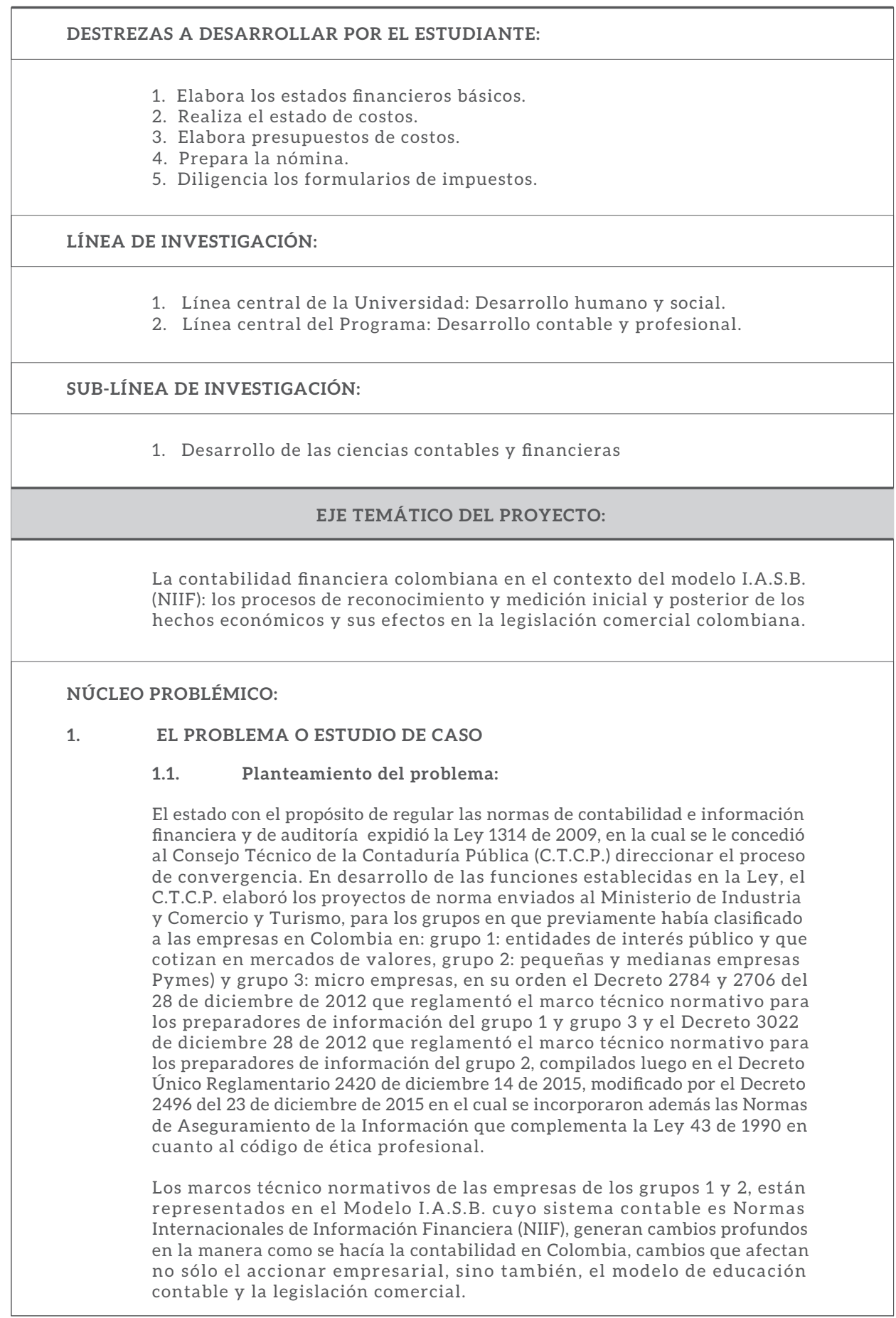


Tabla 5.

\section{Proyecto de aula $2^{\circ}$ semestre}

\section{2 . \\ Formulación del problema.}

¿Cuáles son los aspectos diferenciadores en el reconocimiento y medición de los hechos económicos en el contexto de las NIIF para Pymes para efectos de presentación de la información financiera a usuarios externos e internos?

\subsection{Sistematización del problema}

1.3.1. ¿Cuáles son los aspectos diferenciadores en las mediciones contables de las sociedades por acciones y de personas en el contexto de las NIIF para Pymes?

1.3.2. ¿Cómo se efectúa el reconocimiento y medición de los instrumentos financieros básicos?

1.3.3. ¿Cuáles son los criterios establecidos en la sección 28 de la NIIF para Pymes: Beneficios a los empleados para el reconocimiento y medición contable?

1.3.4. ¿Qué tipos de empresas pueden aplicar el sistema de costos por órdenes de producción y cómo se efectúa el proceso de acumulación de los costos?

1.3.5. ¿Cuáles son los procedimientos para aplicar, contabilizar, calcular y analizar los gastos indirectos de fabricación y sus variaciones?

1.3.6. ¿Cómo se determina el costo por el modelo de costeo variable y cuál es su aplicación en la toma de decisiones?

1.3.7. ¿Qué atributos de la legislación comercial no se contemplan en la Sección 22 de la NIIF para Pymes?

1.3.8. ¿Cuáles son las diferencias, tipos, clases y formas sociales establecidas en la legislación colombiana y las obligaciones de los asociados según la clase de sociedad?

2 .

\section{OBJETIVOS:}

2.1. Objetivo general.

Determinar los aspectos diferenciadores en el reconocimiento y medición de los hechos económicos en el contexto de las NIIF para Pymes para efectos de presentación de la información financiera a usuarios externos e internos

\subsection{Objetivos específicos.}

\section{CONTRIBUCIÓN DESDE LA ASIGNATURA PROCESOS CONTABLES A TRAVÉS DE TRES (3) A.B.P.}

2.2.1. Identificar los aspectos diferenciadores en las mediciones contables de las sociedades por acciones y de personas establecidas en la Sección 22 de la NIIF para Pymes respecto de la legislación societaria en Colombia.

2.2.2. Efectuar el reconocimiento y medición de los instrumentos financieros básicos contenidos en la Sección 11 de la NIIF para Pymes para establecer diferencias con el modelo de contabilidad simplificada.

2.2.3. Especificar los criterios establecidos en la sección 28 de la NIIF para Pymes: Beneficios a los empleados para su reconocimiento y medición contable.

\section{CONTRIBUCIÓN DESDE LA ASIGNATURA COSTOS PARA LA TOMA DE DECISIONES A TRAVÉS DE TRES (3) A.B.P.}

2.2.4. Identificar los tipos de empresas donde se puede aplicar el sistema de costos por órdenes de producción para efectuar el proceso de acumulación de los costos.

2.2.5. Describir los procedimientos necesarios para aplicar, contabilizar, calcular y analizar los costos indirectos de fabricación y sus variaciones en los diferentes tipos de empresas.

2.2.6. Calcular los costos de producción por el modelo de costeo variable para efectos toma de decisiones empresariales. 
Tabla 5.

Proyecto de aula $2^{\circ}$ semestre

\section{CONTRIBUCIÓN DESDE LA ASIGNATURA LEGISLACIÓN SOCIETARIA A TRAVÉS DE TRES (3) A.B.P.}

2.2.7. Identificar los aspectos de la legislación comercial que no se contemplan en la Sección 22 de la NIIF para Pymes.

2.2.8. Establecer las diferencias, tipos, clases, formas sociales y obligaciones de los asociados según el tipo de sociedad establecidas en la legislación colombiana.

3.

\section{JUSTIFICACIÓN}

La Ley 1314 de 2009 modificó el sistema contable colombiano al converger la información financiera con base en el Decreto 2649 de 1993 a Normas Internacionales de contabilidad e información financiera. Esto trajo como consecuencias criterios para el reconocimiento y medición de los hechos económicos basados en principios y no en reglas.

Dentro de esta dinámica se presentan cambios estructurales en el proceso contable, respecto del reconocimiento y medición de las transacciones financieras, al pasar de una medición al costo histórico como estaba establecido en el Decreto 2649 de 1993 a mediciones a costo o valor razonable dependiendo de la naturaleza de la transacción.

Motiva el desarrollo del proyecto de aula ahondar en el conocimiento de esas diferencias entre el modelo NIIF y el modelo PCGA locales que conlleven a una comprensión adecuada de la norma a fin de presentar información financiera fiable y relevante.

4.

\section{METODOLOGÍA.}

En la Universidad del Sinú - Elías Bechara Zainúm, el proyecto de aula se desarrolla en 5 fases detalladas a continuación:

\subsection{Fase 1: descripción del problema o estudio de caso}

Se describen todas las características de la problemática, los hechos y los acontecimientos que están insertos en el problema, se mencionan los antecedentes del problema, los objetivos y sus relaciones con los A.B.P. que se deben desarrollar por asignaturas. En esta fase los estudiantes construyen los problemas de los respectivos A.B.P. de las asignaturas articuladas al proyecto docente, antecedentes y objetivos.

\subsection{Fase 2: formación de los equipos de trabajo.}

El docente líder para formar los equipos emplea las técnicas de agrupamiento al azar o permitiendo la agrupación por afinidad o agruparlos de tal modo que aquellos con más conocimiento de la temática apoyen a los demás estudiantes. Los grupos no deben exceder de 8 ni pueden ser inferiores a 4 . En esta fase el docente explica la forma de organizar el trabajo independiente del estudiante, a qué fuentes acudir, y la asignación de las responsabilidades de cada integrante.

\subsection{Fase 3: planificación y organización del trabajo.}

En esta fase el docente líder con su equipo de apoyo realizan dos actividades básicas: 1.- dar las pautas para la planificación del trabajo de los estudiantes, es decir, la elaboración del plan de actividades en el desarrollo de los A.B.P. de los estudiantes, y 2.- distribuir los roles del trabajo de los equipos que les permita el trabajo con comodidad y con conciencia de las responsabilidades individuales en el trabajo. 
Tabla 6.

\section{Organización del trabajo}

\section{Coordinador}

Dirige y organiza el trabajo de campo, promueve la participación de todos.

\section{Responsable de materiales}

Garantiza el material necesario para realizar el trabajo.
Relator

Organiza y recopila la información y la socializa con el grupo.

\section{Protocolante}

Toma nota de todas las actividades realizadas por el grupo y socializa las conclusiones del relator.

Fuente: Elaboración propia

4.4. Fase 4: ejecución del proyecto.

Esta fase es desarrollada por los estudiantes, quienes haciendo uso de la herramientas entregadas por los docentes para realizar el trabajo de indagación, realizan el trabajo de campo que les permite elaborar sus A.B.P. que son el producto de la fase de ejecución del proyecto.

\subsection{Fase 5: evaluación.}

El docente líder con los docentes de apoyo evalúan el trabajo investigativo de los estudiantes, a partir de las competencias genéricas y específicas adquiridas en el desarrollo de sus A.B.P.

\section{EVALUACIÓN DEL PROYECTO}

El proceso de evaluación del trabajo realizado por los estudiantes, le permite a los docentes tomar decisiones acerca de la forma como el estudiante construye su propio conocimiento, y como ésta se realiza en todas las fases del proyecto, la evaluación es continua basada en observaciones reflexivas del accionar del estudiante en las tareas que debe realizar en sus A.B.P (Agudelo, González \& Osorio, 2007).

El proceso de evaluación se realiza en tres momentos:

1. Dentro de las primeras seis (6) semanas del período académico se evalúan tres (3) .A.B.P. uno por cada asignatura que articula el proyecto de aula.

2. Una segunda evaluación entre la séptima (7a.) y once (11) del período académico, con base en los A.B.P. de las asignaturas de articulan el proyecto.

3. Y una tercera evaluación realizada a los A.B.P. desarrollados por los estudiantes entre las semanas trece (13) y diez y seis (16) del período.

Los docentes retroalimentarán permanentemente las evaluaciones. La evaluación durante el período académico se distribuirá así: 
Tabla 7.

Evaluación del proyecto de aula.

\begin{tabular}{|c|c|c|c|c|}
\hline \multicolumn{5}{|c|}{ DISTRIBUCIÓN DE LA EVALUACIÓN DEL PROYECTO DE AULA } \\
\hline Cortes & $\begin{array}{c}\text { \% A.B.P. } \\
\text { Procesos contables }\end{array}$ & $\begin{array}{l}\text { \% A.B.P. } \\
\text { Costos para toma } \\
\text { de decisiones }\end{array}$ & $\begin{array}{l}\text { \% A.B.P. } \\
\text { Legislación } \\
\text { societaria }\end{array}$ & TOTAL \\
\hline Primer & $10 \%$ & $10 \%$ & $10 \%$ & $30 \%$ \\
\hline Segundo & $10 \%$ & $10 \%$ & $10 \%$ & $30 \%$ \\
\hline Tercer & $10 \%$ & $10 \%$ & $10 \%$ & $30 \%$ \\
\hline TOTALES & $30 \%$ & $30 \%$ & $30 \%$ & $90 \%$ \\
\hline \multicolumn{4}{|c|}{$\begin{array}{l}\text { 1. Pertinencia de la recolección y tabulación de información (bibliográfica) } \\
\text { 2. Coherencia y profundidad en la redacción de textos, uso de normas A.P.A. } \\
\text { 3. Presentación de los avances y finales de los textos desarrollados en los .A.B.P. } \\
\text { 4. Sustentación de sus trabajos de A.B.P }\end{array}$} & $\begin{array}{l}\text { a) } \\
\text { P.A. } \\
\text { B.P. }\end{array}$ \\
\hline
\end{tabular}

Tabla 8.

Cronogramas

\begin{tabular}{|c|c|c|c|c|c|c|c|c|c|c|c|c|c|c|c|c|}
\hline \multicolumn{17}{|c|}{ CRONOGRAMA DEL PROYECTO: } \\
\hline \multicolumn{17}{|c|}{ CRONOGRAMA DE ACTIVIDADES } \\
\hline \multirow{2}{*}{ Periodos / Actividades } & \multicolumn{4}{|c|}{ 1er. Mes } & \multicolumn{4}{|c|}{ 2do. Mes } & \multicolumn{4}{|c|}{ 3er. Mes } & \multicolumn{4}{|c|}{ 4to. Mes } \\
\hline & 1 & 2 & 3 & 4 & & 2 & 3 & 4 & 1 & 2 & 3 & 4 & 1 & 2 & 3 & 4 \\
\hline \multicolumn{17}{|l|}{ Descripción de problemas } \\
\hline \multicolumn{17}{|l|}{ Formación de los equipos } \\
\hline \multicolumn{17}{|l|}{ Planificación del trabajo } \\
\hline \multicolumn{17}{|l|}{ Recolección de información } \\
\hline \multicolumn{17}{|l|}{ Revisiones del trabajo } \\
\hline \multicolumn{17}{|l|}{ Entregas de A.B.P. } \\
\hline Producto fipah horama Econór & , Vo & 27 & & & & 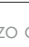 & 20 & $t_{1}$ & $8-30$ & & & & & & & \\
\hline
\end{tabular}

Fuente: Elaboración propia 


\section{CONCLUSIONES}

El proyecto de aula debe entenderse como una estrategia utilizada por el docente para desarrollar conocimiento complejo en el estudiante y en la cual no sólo aprende el estudiante, sino que el ejercicio de la docencia se ve fortalecido, convirtiéndose en un proceso de doble vía.

Del mismo modo, la articulación de la metodología del A.B.P. como estrategia pedagógica genera muchos beneficios para el estudiante en su proceso de autoaprendizaje en razón al desarrollo de las estructuras de monitoreo y control permanente del docente. El desarrollo de A.B.P. se hace más relevante para el caso de asignaturas disciplinares de la profesión contable. Así, el estudiante asume el A.B.P. como una actividad propia y necesaria en su proceso de aprendizaje.

Con respecto de las obligaciones de los docentes, entre ellas se encuentran la presentación de productos publicables por asignaturas desarrolladas, siendo el proyecto de aula una actividad inherente al proceso de formación, en la articulación de cada proyecto de aula y dependiendo de las disciplinas que convergen en su desarrollo, se podrán elaborar los productos a partir de la contribución que desde cada asignatura se hace al proyecto de aula. Lo anterior permite la elaboración de manuscritos o ensayos por cada uno de los docentes que aportan al proyecto de aula.

\section{REFERENCIAS BIBLIOGRAFICAS}

Agudelo, E., González, M., \& Osorio, M. (2007). Un modelo de evaluación curricular alternativa y pertinente con el diseño curricular basado en la solución de problemas para la formación de profesionales: caso Programa de Contaduría Pública de la Universidad de Antioquia. Revista Contaduría Universidad de Antioquia; Jul-Dic; 51.

Bruning, R. H., Schraw, G. J., Norby, M. \& Ronning, R. R. (2004). La Psicología Cognitiva y la instrucción. (4a. ed.). Upper Saddle River, NJ: Merrill/Prentice Hall.

Cerda, H. (2003). Pedagogía de proyectos: algo más que una estrategia. Revista Magisterio, Educación y Pedagogía. Vol. 2, Santa fe de Bogotá.

Chaparro, C. (2003). Proyectos de aula vs homogeneización. Revista Magisterio, Educación y Pedagogía. Vol. 2, Santa fe de Bogotá.

Deleuze, G. \& Guattari, F. (2001). ¿Qué es la Filosofía? Barcelona, Anagrama.

Farías, G. \& De Agüero, M. (2014). Innovación en la práctica docente en contabilidad. Revista Criterio Libre No. 21 Bogotá (Colombia). Edición Julio-Diciembre 2014. (Pp. 249-262).

Instituto Tecnológico y de Estudios Superiores de Monterrey (2018). El Aprendizaje Basado en Problemas como técnica didáctica. México. Recuperado de http://www.sistema.itesm.mx/va/dide/inf-doc/estrategias/ 
Londoño, Pilar y Calvache, J. (2010). Las estrategias de enseñanza: aproximación teórico-conceptual. Editorial Kimpres Ltda. Bogotá.

Martínez, G. (2008). La educación contable: encrucijada de una formación monodisciplinaria en un entorno complejo e incierto. Editorial Universidad del Cauca. Popayán (Colombia).

Ministerio de Educación Nacional, (2002). Decreto 0808 de abril 25. Recuperado de http://www.mineducacion.gov.co.

Moust, J. Bouhuijs, P. y Schmidt, H. (2007). El aprendizaje basado en problemas: guía del estudiante. Gráficas Cuenca.

Ortiz, D. (2015). El constructivismo como teoría y método de enseñanza. Ecuador. Recuperado de http://www.redalyc.org/articulo.oa?id=44184609600

Porlan, R. (1990). El maestro como investigador en el aula. Investigar para conocer, conocer para enseñar. Revista Investigación en la Escuela $N^{\circ}$ 1, Sevilla.

Rincón, G. (2012). Los proyectos de aula y la enseñanza y el aprendizaje del lenguaje escrito.

Schunk, D. H. (2012). Teorías del Aprendizaje. México: Pearson. VI Edición.

Silva, J. y Maturana, D. (2017). Una propuesta de modelo para introducir metodologías activas en educación superior. México D. F. En Revista Innovación educativa.

Tobón, S., Pimienta, J. y García, J. (2010). Secuencias didácticas: aprendizaje y evaluación de competencias. México. D. F. Editorial Pearson educación

Tudge, J. \& Scrimsher, S. (2003). Lev S. Vygotsky sobre la educación: un enfoque histórico-cultural, interpersonal e individual al desarrollo. En B. J.Zimmerman y D. H. Schunk (Eds.), pp. 207-228.

Universidad del Sinú - Elias Bechara Zainum (2003). Proyecto educativo Institucional, Acuerdo 004 de febrero 25. Montería. Recuperado de http://wwww.unisinu.edu.co.

Vieira, T. (2003): El aprendizaje significativo de Ausubel: algunas consideraciones desde el enfoque histórico cultural. Universidades. (26), 37-43. Recuperado http://redalyc.uaemex.mx/redalyc/pdf/373/37302605.pdf.

Vizcarro, C. \& Juárez, E. (2008). ¿Qué es y cómo funciona el aprendizaje basado en problemas? En El aprendizaje basado en problemas en la enseñanza universitaria. ISBN 978-84-8371-778-3, págs. 17-36 Recuperado de http://www.ub.edu/dikasteia/LIBRO_MURCIA.pdf 